\title{
DNA Sequencing: Current State and Prospects of Development
}

\author{
Lusine Gasparyan"1,2, Ilya Mazo1, Vahan Simonyan³, Ferdinand Gasparyan1,2 \\ ${ }^{1}$ Argentys Informatics, LLC, Gaithersburg MD, USA \\ ${ }^{2}$ Yerevan State University, Yerevan, Armenia \\ ${ }^{3}$ The Department of Biochemistry \& Molecular Medicine, The School of Medicine and Health Sciences, The George Washington \\ University, Washington, DC, USA \\ Email: fgaspar@ysu.am
}

How to cite this paper: Gasparyan, L., Mazo, I., Simonyan, V. and Gasparyan, F. (2019) DNA Sequencing: Current State and Prospects of Development. Open Journal of Biophysics, 9, 169-197.

https://doi.org/10.4236/ojbiphy.2019.93013

Received: May 5, 2019

Accepted: July 14, 2019

Published: July 17, 2019

Copyright (c) 2019 by author(s) and Scientific Research Publishing Inc. This work is licensed under the Creative Commons Attribution International License (CC BY 4.0).

http://creativecommons.org/licenses/by/4.0/

\begin{abstract}
In this review, we collected and classified the stages of development of DNA sequencing methods and described its peculiarities. We pay attention mostly on solid-stead nanopore sequencing methods. Detailed discussion of the peculiarity and feasibility of the electrical methods of DNA sequencing is discussed. The detail analyses of the literature data, some critical considerations and the potential ways of optimization of DNA nanopore sequencing were presented.
\end{abstract}

\section{Keywords}

DNA Sequencing, ISFET Sensor, EIS Sensor, Solid-Stead Nanopore

\section{Introduction}

Since the discovery of the double-helix structure of the Deoxyribose Nucleic Acid (DNA) [1], the research on DNA sensors has grown drastically. Biosensors, especially DNA-biosensors are utilized to study DNA-related phenomena. The process of DNA sequencing is a precise determination of the amount and distribution of the nucleotides (A-adenine, T-thymine, C-cytosine, and G-guanine) in DNA molecules. Quick improvements in the cost and speed of DNA sequencing are having a strong influence on comprehensive genome research. Genomic information has many applications beside human medicine, for example, in security (DNA identification, pathogens detection, consumables authentication), insurance (individual polices, risk assessment), biotech (microbial engineering, GMO, livestock breeding, agriculture), health care (diagnosis, disease prevention, pharmacogenetics, gene therapy, vaccines, transplant programs), 
defense, and evolutionary biology. However, the costs of sequencing are still too high for routine application. As noted in [2] [3] single-molecule genome sequencer using solid-state nanopore devices is one of the most promising candidates for a realization of $\$ 1000$ personal genome-sequencing.

During its development DNA sequencing has some stages. Evolution of first, second, third and fourth generation of sequencing platforms and their applications detailed are presented in [3] [4] [5] [6]. One of the challenges for next-generation DNA sequencing is the creation of reliable, stable, reproducible, and cheap nanoscale structures-devices. Several nanoscale electronic methods have been proposed for high-throughput single-molecule nucleic acid sequence identification. DNA bases can be identified statistically in nanopore translocation events. Under the influence of an electric field, the negatively charged polynucleotides can be captured and drawn through the channel in a process termed "translocation".

In this review, we collected and classified the stages of development of DNA sequencing methods and described its peculiarities. We pay attention mostly on solid-stead nanopore sequencing methods. Detailed discussion of the peculiarity and feasibility of the electrical methods of DNA sequencing is discussed. The detail analyses of the literature data, some critical considerations and the potential ways of optimization of DNA nanopore sequencing were presented. In the contrast of other reviews in this field, we try to more critically discuss the "weak points" of some works (see Part 4).

\section{DNA Sequencing Methods}

DNA sequencing methods are:

$\checkmark$ Basic DNA sequencing

- Sanger method (chain termination). Sanger sequencing is a method DNA sequencing first commercialized by Applied Bio systems, based on the selective incorporation of chain-terminating dideoxynucleosides by DNA polymerase during vitro DNA replication [7] [8].

- Maxam-Gilbert method (chemical termination). This method is based on nucleobase-specific partial chemical modification of DNA and subsequent cleavage of the DNA backbone at sites adjacent to the modified nucleotides [9].

$\checkmark$ Advanced DNA sequencing

- Shotgun method. Shotgun sequencing is a method used for sequencing long DNA strands [10].

$\checkmark$ Next generation DNA sequencing

- Illumina sequencing method. This sequencing method is based on reversible dye-terminators that enable the identification of single bases as they are introduced into DNA strands [11].

- Pyrosequencing method. Pyrosequencing is a method of DNA sequencing based on the "sequencing by synthesis" principle, in which the sequencing is 
performed by detecting the nucleotide incorporated by a DNA polymerase [12].

$\checkmark$ Solid-state (and biological) sequencing method [13].

Further our main attention will be focusing in last solid-state (and biological) nanopore sequencing method.

Although sequencing methods using nanopores have been studied in some detail, nevertheless, new perceptual architectures are emerging that are intended for the next generation of biosensors. In recent years many types of architectures have been proposed for new approaches to biomolecular sensing using nanoelectronics, including also the appearance of molecular (tunneling) junctions as a platform for perception.

In the past decade, the single-nanometer-scale pores demonstrated great capability for the detection, identification, and characterization of a variety of analytes, such as biomolecules like bacteria [14], protein [15] [16] [17] [18] [19], antibody [20] [21], nucleic acid [22] [23], DNA [24] [25], RNA (Ribonucleic acid) [26] [27].

Now the bio-, graphene, nanoribbon, nanotube, solid-state and hybrid nanopores are used successfully.

A large number of researches have been reported on DNA sequencing with solid-state nanopores based on different substrates, such as silicon oxide $\left(\mathrm{SiO}_{2}\right)$ [28] [29], silicon nitride $\left(\mathrm{SiN}_{x}\right)$ [30] [31] [32] [33], aluminum oxide $\left(\mathrm{Al}_{2} \mathrm{O}_{3}\right)$ [34] [35], molybdenum sulfide $\left(\mathrm{MoS}_{2}\right)$ [34] [36] [37] [38] [39] [40], boron nitride (BN) [41] [42] [43], graphene [20] [37] [38] [44]-[52], carbon nanotube [53] [54] [55] [56] [57], nanochannel [40] [58] [59] [60] and polymers [61] [62] [63] [64] [65].

Solid-state nanopores exhibited remarkable chemical, thermal, and mechanical stability and extraordinary versatility in terms of the size, shape, and surface properties, as well as structural robustness. Different types of nanopores and typical technologies applied in the field of solid-state nanopore-based DNA sequencing have been detailed analyzed and reviewed in [4] [61] [66]. Nanopore sequencing is a label-free and amplification-free single-molecule approach, in which DNA molecules are driven through channels, producing signals that allow researchers to identify the corresponding sequences [62] [63] [67]-[76].

Nanopore-based DNA sequencing has led to fast and high-resolution recognition and detection of DNA bases. Solid-state and biological nanopores have low signal-to noise ratio $(\mathrm{SNR})(<10)$ and are generally too thick $(>5 \mathrm{~nm})$ to be able to read at single-base resolution. A nanopore in graphene has a SNR of $\sim 3$ under DNA ionic current. Using atomistic and quantum simulations, it is found that a single-layer $\mathrm{MoS}_{2}$ is an extraordinary material (SNR > 15) for DNA sequencing by two competing technologies (nanopore and nanochannel) [40]. To increase both SNR and dwell time in a conventional silicon-substrate nanopore, are added nano-well of $100-150 \mathrm{~nm}$ in diameter [77]. $\mathrm{A} \mathrm{MoS}_{2}$ nanopore shows four distinct ionic current signals for single-nucleobase detection with low noise level. In addition, a single-layer $\mathrm{MoS}_{2}$ shows a characteristic change in the total 
density of states for each base. The direct band gap of $\mathrm{MoS}_{2}(1.88 \mathrm{eV}$ [78]) is significantly changed when bases are placed on top of the pristine $\mathrm{MoS}_{2}$ and armchair $\mathrm{MoS}_{2}$ nanoribbon, thus making $\mathrm{MoS}_{2}$ a promising material for base detection via transverse current tunneling measurements [36] [40]. Molecular dynamics simulations protein transport a nanopore in a quasi-2D heterostructure stacked together by graphene and $\mathrm{MoS}_{2}$ nanosheets proposed in [37] [38]. In the case of a weak signal current, there is a need of its amplification. To increase the measured signal current in various complex systems, the movement of DNA must be slowed down. To do this, one suggests using a protein nanopore [24] for example, in combination with solid-state nanopores. It is shown that a protein nanopore with a covalently attached adapter molecule can continuously identify unlabeled nucleoside 5'-monophosphate molecules with accuracies averaging $99.8 \%[19]$.

The data [79] show that near-infrared SERS (surface-enhanced Raman scattering) on colloidal silver clusters also is an effective method of detecting single molecules. It is applicable to a wide range of molecules, including "colorless" biomolecules, for example, nucleotides in DNA sequencing.

The technique of reproducible conductivity measurements on single nucleotides with limited conformation and an improved algorithmic approach for isolating nucleotide bases is proposed in the [80]. The method of quantum point contact single-nucleotide conductance sequencing uses combed and electrostatically connected single DNA and RNA nucleotides on a self-assembled monolayer of cysteamine molecules. It is shown that by changing the applied bias and the $\mathrm{pH}$ condition, it is possible to turn on and turn off the molecular conductivity, which leads to a reversible nucleotide perturbation for electronic recognition. In [80], the potential of using simple surface modifications and existing biochemical fragments in individual nucleotide bases for reliable, direct, single-molecular nanoelectronics DNA and a method for identifying nucleotides of RNA for sequencing is demonstrated.

Drawing attention to the timely need for standardization of reliable nanosize devices for cheap, fast and scalable DNA detection, in [44] the nanoscale formed by the side heterostructure of graphene and hexagonal boron nitride is considered as a potential architecture. It is noted that blocks of DNA structure, nucleotide groups unambiguously associated with nano-gang states and emerging induced states, can be attributed as leaving a DNA sequence impression in the calculated current-voltage characteristic. Two offset windows are advanced: lower $(1-1.2 \mathrm{~V})$ and higher $(2.7-3 \mathrm{~V})$, where it is possible to uniquely identify all four bases from the current traces, although a higher sensitivity is obtained in the higher voltage window. Ways to improve the sensitivity of the DNA-nucleotide base using functionalized graphene nanogap as a solid-state device was analyzed in [45]. The appropriate translocation rate of the base molecule provides a time-dependent function of interaction change inside of interaction interval of each individual base with graphene nanopore. The forces between bases and graphene nanopore are calculated as interaction characteristics 
of bases [47]. Molecular dynamics method is used for the DNA base and graphene nanopore calculations. The time-dependent in-plane for graphene transient force signal resolution for different bases is probed. Possibility of base identification by combination of transient in-plane force taken as orientation averaged signal is studied. The nucleobases are inserted into a pore in a graphene nanoribbon, and the electrical current and conductance spectra are calculated as functions of voltage applied across the nanoribbon. The conductance spectra and charge densities are analyzed in the presence of each nucleobase in the graphene nanopore. The results indicate that due to significant differences in the conductance spectra the proposed device has adequate sensitivity to discriminate between different nucleotides. Moreover, it is shown that the nucleotide conductance spectrum is affected little by its orientation inside the graphene nanopore.

Since DNA bases are different from each other in atomic scale, it is essential to collect base-specific information at atomic level to correspond to the DNA sequence with the measured signals. The amount of current which can pass through the nanopore at any given moment therefore varies depending on whether the nanopore is bonded by an A, a C, a G or T nucleotides. The change in the current through the nanopore as the DNA molecule passes through the nanopore represents a direct reading of the DNA sequence. In this case, four separate reading would be used, each one responsible for identifying one of the four DNA bases. The transient characteristics of the individual bases can be used for identification of the bases.

According to the different types of the signals, the DNA detection methods can be roughly classified into two categories [81]:

- the electrical detection methods, and

- the optical readout methods.

The optical detection methods require excitation irradiation source and photo-detector that transforms fluorescence light into measurable electrical signal, and optoelectronic connection to the reaction chamber. Therefore, the optical systems are often relatively large and expensive to be used in hand-held point-of-care devices [82].

In this review, optical detection methods will not be considered.

\section{Electrical Detection Methods}

Electrical detection methods for nanopore-based DNA sequencing are:

- Method Based on Ionic Blockade Current [83] [84] [85] [86] [87];

- Method Based on Tunneling Current [35] [82] [88] [89] [90] [91];

- Method Based on Capacitance Variation [92] [93] [94];

- Method Based on Electric Potential Change [95] [96].

\subsection{Ionic Blockade Current Method}

The current blockades are found to be sensitive to the properties of the nucleo- 
tide composition, sizes, and secondary structure, and to physical parameters such as the driving field intensity, temperature, and ionic strength of the bio-liquid. These blockades are therefore a rich and powerful source of information regarding the dynamics of polynucleotides in the pore. The process of translocation occurs in two main stages (Figure 1):

- nucleotide "capture" in which one of the nucleotide's ends is threaded a small distance through the channel, and

- nucleotide sliding through the channel.

During translocation, the ionic current flowing through the channel is mostly blocked, indicating the presence of the polymer/molecule inside the channel.

In 1996, Kasianowicz et al. opened a whole new field when they reported the first ionic current blockades caused by the translocation of single-stranded RNA and DNA homopolymers through a $1.4 \mathrm{~nm}$ wide, $10 \mathrm{~nm}$ long, channel-forming $\alpha$-hemolysin $(\alpha-\mathrm{HL})$ in an artificial lipid bilayer [97] .

The experimental and theoretical efforts to elucidate polymer capture and the transport dynamics of biopolymers in nanosized pores are detailed reviewed in [65]. The initial concept for nanopore sequencing involved threading an individual single-stranded DNA (ssDNA) molecule through the staphylococcal $\alpha$-HL protein pore under an applied potential while recording modulations of the ionic current passing through the pore. Each base will be registered, in sequence, by a characteristic decrease in current amplitude [87] [97] [99] [100] [101]. It has been demonstrated that an individual nucleobase can be identified on a static strand in a nanopore [102]; however, the rate of DNA translocation under a potential is too high for the necessary current resolution in a moving strand unless the bases are subjected to chemical modification with bulky groups [103]. The main technical problems required for the monophosphatization of high-resolution nucleosides are presented in [19]. It is shown that the robustness of lipid-bilayers is very sensitive to external and experimental conditions, to $\mathrm{pH}$ value, salt composition, temperature and mechanical stress, which can make the supporting membrane very unstable.

When the pore is empty the path of the ions in bio-liquid is not blocked and relatively large ion current flows through membrane, when the polymer/DNA

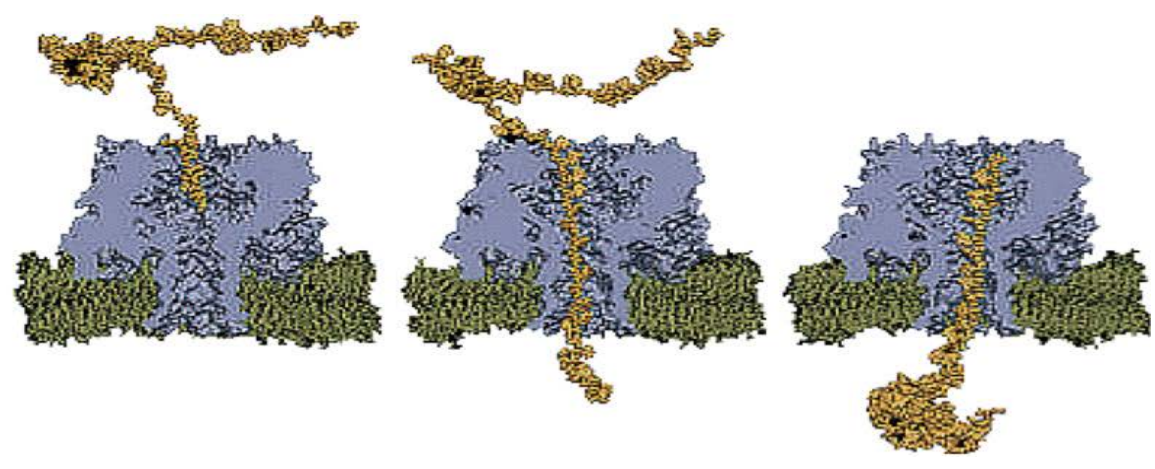

Figure 1. Molecular dynamic simulation of a ssDNA molecule translocating from one side of the membrane (pore) to the other. Adapted from Dekker's review [98]. 
enters the pore, it partially blocks the path of the ions, resulting in a significant decrease in current (Figure 2). When a molecule is translocate through a nanopore that partially blocks and prevents ions from freely flowing from one side to the other, and thus modifies the ionic transport. Despite that DNA also carries a charge through the pore, its relative speed compared to translocation of ions is very slow and this translates into a "blockade event", during which the ionic current briefly exhibits a lowered value. These translocation events give rise to spikes, also named pulses, in the ionic current baseline that can be characterized by their frequency, dwell time, and drop amplitude. The polymer/DNA makes many attempts to cross the pore; the unsuccessful attempts result in current blockades of very short durations ( $t_{0}$ in Figure 2 ).

The modulation of current flowing through a nanowire by DNA molecules has been proposed as high throughput sequencing techniques. Xie et al. successfully detected the translocation of DNA molecules through a nanopore drilled at the edge of a silicon nanowire connected with electrodes [104]. Using such a nanowire-nanopore field-effect transistor (FET) sensor, they demonstrated that localized changes in the electrical potential during DNA translocations are responsible for FET conductance decreases (modulation) that correlated with the ionic current blockades.

The full details of the current blockade phenomenon are extremely complex: the involved variables are the pore size, pore geometry, DNA dynamics, homogeneity of the bio-liquid, chemical associations and charge condensations on the pore, ionic conditions on both sides of the separating membrane (including the ionic cloud that may condense around the pore), concentration of the macromolecules in the solution, ionic condensation on the macromolecules, voltage difference across the pore and temperature.

In general the translocation process can be viewed as a process controlled by following main factors:

- external driving forces (gravity, electrophoretic and drag forces),

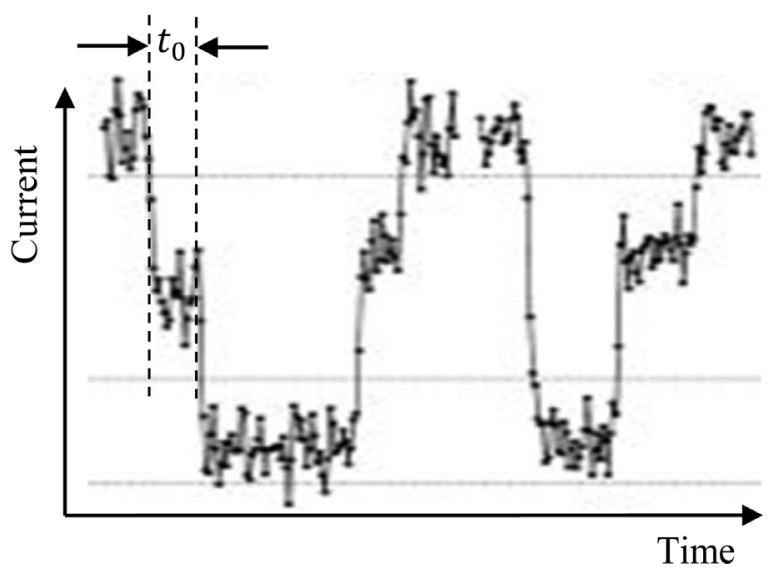

Figure 2. Example of ionic current traces illustrating the substrate structure of polynucleotide-induced current blockade events [87]. 
- applied potentials to lateral electrodes,

- polymer dynamics in the aqueous solution,

- type, concentration and orientation of nucleotides,

- pore or channel architecture (form, sizes), and

- molecule-pore interactions in atomic scale.

The main part of stages of translocation dynamics through the pore are detailed analyzed in [64]. It is provided a combined theory-experiment progress report on the understanding of (bio)polymer translocation.

For ionic conductance $G$ of a cylindrical nanopore the following expression is commonly used in the literature [105]:

$$
G=\sigma\left(\frac{4 l}{\pi d^{2}}+\frac{1}{d}\right)^{-1}
$$

Here $\sigma$ is the bulk conductivity, $l$ is the length (the membrane thickness) and $d$ is the diameter of the nanopore.

The capture radius $r_{c}$ is the distance at which the electrophoretic force overwhelms the free thermodynamic diffusion and makes unlikely a molecule to escape the electric field forced diffusion (Figure 3 ). Once within the capture radius, single molecules are eventually captured and translocate to the other side. Molecules are captured by open bonds of hydrogen, nitrogen or carbon atoms of nucleotides (usually weak bonds) and move under the action of electroosmotic forces in the electrolyte.

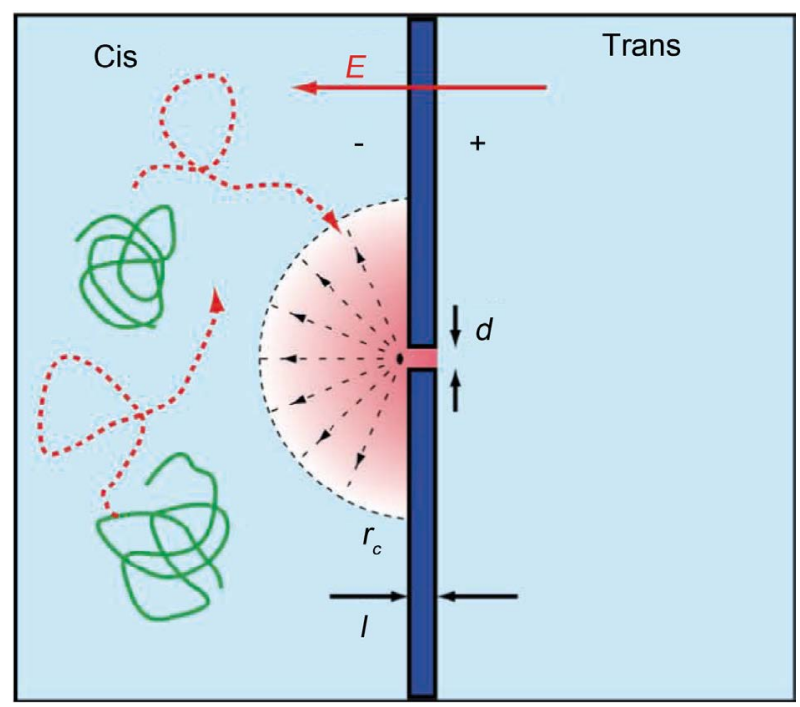

Figure 3. Scheme of capture of biopolymer in a nanopore. When an electric field is applied across a membrane, the potential drop mainly occurs at the nanopore, forming a funneling field driving the molecule toward the pore aperture. Outside of the capturing radius $r_{c}$, the electric field plays a marginal role and the molecules freely diffuse. Picture adapted from [3]. "Cis" indicates that the functional groups are on the same side of the carbon chain, while "Trans" conveys that functional groups are of opposing sides of the carbon chain. 


\subsection{Tunnel Current Method}

As one of potential future techniques mentioned in [6] is DNA sequencing by tunneling currents. Besides the measurement of the changes in the ionic current, as the DNA passes through the pore, there are efforts to measure tunnel current flow through each base of DNA molecule (in a technique called Tunneling Currents DNA Sequencing) as it traverses the pore or channel. The expectations are that each base will show sufficiently different tunneling current and the base sequence will be deduced from the differences in the tunneling currents amplitude. This technique could have the potential to sequence orders of magnitude faster than the ionic current methods [6]. As noted yet the graphene nanogaps and nanopores also show potential for electrical DNA sequencing [46]. The change in the electric tunnel current is determined as a function of the applied bias and the coupled differential conductivity is analyzed at a voltage that seems to be suitable for distinguishing four nucleotides. A negative effect of differential resistance for one of the nucleotides (deoxyguanosine monophosphate) is predicted. It has been proposed that single molecules of DNA could be sequenced by measuring the physical properties of the bases as they pass through a nanopore [100]. Theoretical calculations suggest that electron tunneling can identify bases in ssDNA without enzymatic processing [106] [107], and it was experimentally shown that tunneling can sense individual nucleotides [108] and nucleosides [109], short DNA oligomers [110]. The use of graphene nanoribbons for transverse current modulation by DNA in two-terminal devices presented in Figure 4, develops in [49]. Usage of the carbon nanotubes (CNT) as tunneling tips presented in Figure 5.

An approach for reading the sequence of a DNA molecule passing between electrodes on a nanopore using tunneling signals associated with a hydrogen bond is proposed in [88]. Interaction with the base electrode is modeled using a scanning tunneling microscope (STM) probe with a nucleobase that is detached from the nucleoside monolayer.

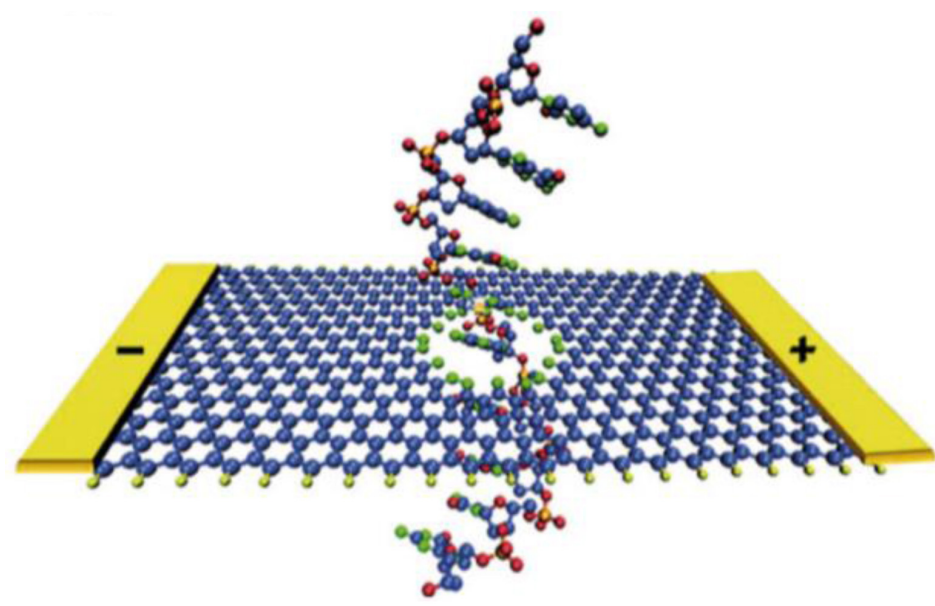

Figure 4. The use of graphene nanoribbons for transverse current modulation by DNA in two-terminal devices [49]. 


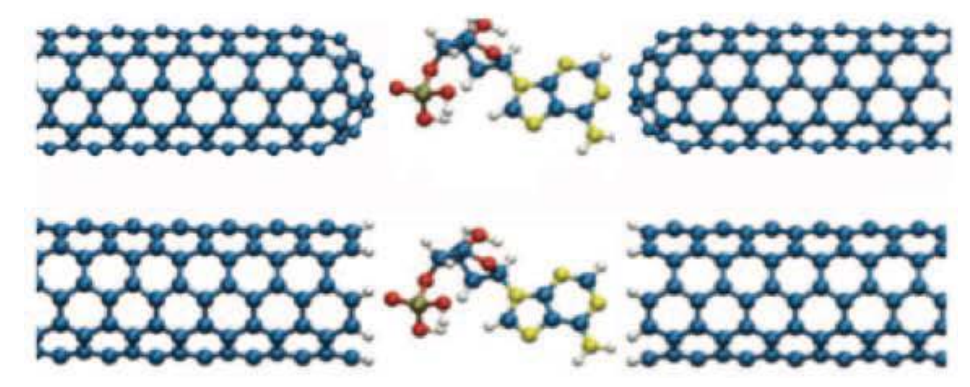

Figure 5. Carbon nanotubes as transverse tunneling electrodes. Two configurations are shown, depending if whether the tips are capped or open (carbon and hydrogen atoms respectively are shown in blue and white). In addition to ideal aspect ratios and unique electronic properties, CNTs may help orient nucleotides in the nanopore through specific $\pi$-stacking interactions. Adapted from [3].

An advantage of transverse tunneling electronic current over ionic current measurements is the fact that the current is measured in the range of nano-ampere, meaning that the translocation speed of DNA is less of a problem than if it is measured at higher frequencies compared to the pico-ampere ionic current of small-diameter nanopores.

Method of mechanically controllable break junction (MCBJ) is successfully used by several authors to investigate translocation behavior of DNA molecules [91] [108] [111]. Schematic of a nanofabricated nano-MCBJ shown on Figure 6. Tsutsui et al. first used sub-2 nm gold nanoelectrodes made from MCBJ technique to detect individual nucleotides in distilled water [108]. With suspended nanogap electrodes in solution, they showed that electron transport occurs by tunneling through nucleotides when freely diffusing individual bases get trapped between the tunneling tips. In such an event, characteristic current pulses are recorded, and they show that the electrical conductance of the bases was significantly different to open the way to individual bases differentiation, with current modulation due to individual bases of about $10 \mathrm{pA}$. In a microchannel, they could similarly detect freely diffusing mononucleotides and short DNA oligomers of up to 22 bases by tunneling measurements.

Method of mechanically controllable break junction (MCBJ) is successfully used by several authors to investigate translocation behavior of DNA molecules [91] [108] [111]. Schematic picture of a nanofabricated nano-MCBJ shown on Figure 6. Tsutsui et al. first used sub-2 $\mathrm{nm}$ gold nanoelectrodes made from MCBJ technique to detect individual nucleotides in distilled water [108]. With suspended nanogap electrodes in solution, they showed that electron transport occurs by tunneling through nucleotides when freely diffusing individual bases get trapped between the tunneling tips. In such an event, characteristic current pulses are recorded, and they show that the electrical conductance of the bases was significantly different to open the way to individual bases differentiation, with current modulation due to individual bases of about $10 \mathrm{pA}$. In a microchannel, they could similarly detect freely diffusing mononucleotides and short 


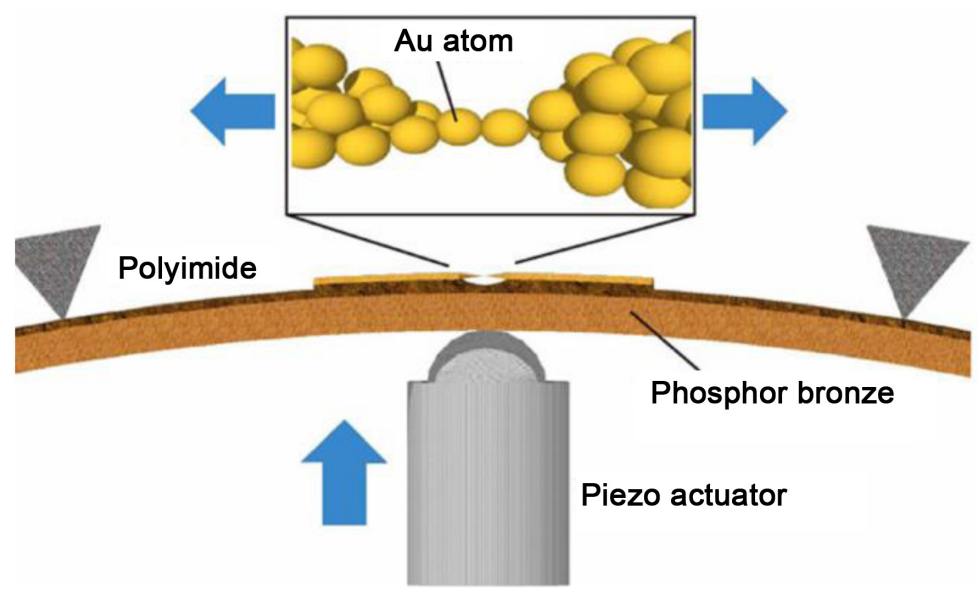

Figure 6. Schematic of a nanofabricated mechanically controllable break junction (MCBJ). Adapted from [91].

DNA oligomers of up to 22 bases by tunneling measurements.

Tunnel current arises in molecular junction created between metallic electrodes and individual nucleobase during passing of the single-nucleobase through nanopore (Figure 7). The change in the electric tunnel current (quantum conductance) is determined as a function of the applied bias and the coupled differential conductivity. As the nucleobase have comparatively high electrical resistance metallic electrodes and nucleobase create a nanosized metal-“dielectric"-metal nanostructure ("instant" molecular junction). Value of tunneling current depends on the length of binding single-nucleobase and characterized it. The appropriate translocation rate of the base molecule provides a time-dependent function of interaction change inside of interaction interval of each individual base with junction electrodes. Issues such as unreliable metal-molecule junction formation, variation of nucleotide conformations, insufficient differences between the molecular orbitals HOMO and LUMO (HOMO-high occupied molecular orbital, LUMO-low unoccupied molecular orbital) are responsible for single-nucleotide conduction lead to overlapping nanoelectronic measurements and poor nucleotide discrimination, especially at low coverage on single molecules. The transient characteristics of the individual bases (i.e. amplitude of the current through junction) can be used for identification of the bases.

Using nano-MCBJ the conductance-time profiles DNA/RNA oligomers are investigated in [111]. Based on the differences in the conductance-time profiles, authors sequentially identified the base-type in the oligonucleotide just passing through the sensing electrode, resulting in the determination of partial sequence. It is proposed a tunneling current based identification as a single-molecule DNA/RNA sequencing. In [91] a single molecule technology that measures the tunneling currents conducted through single base molecules of DNA and RNA passing between two nanoelectrodes of the nano-MCBJ is developed. Nanopores with diameter $\sim 2 \mathrm{~nm}$ are formed on a Si substrate covered with a thin $\mathrm{Si}_{3} \mathrm{~N}_{4}$ film. 


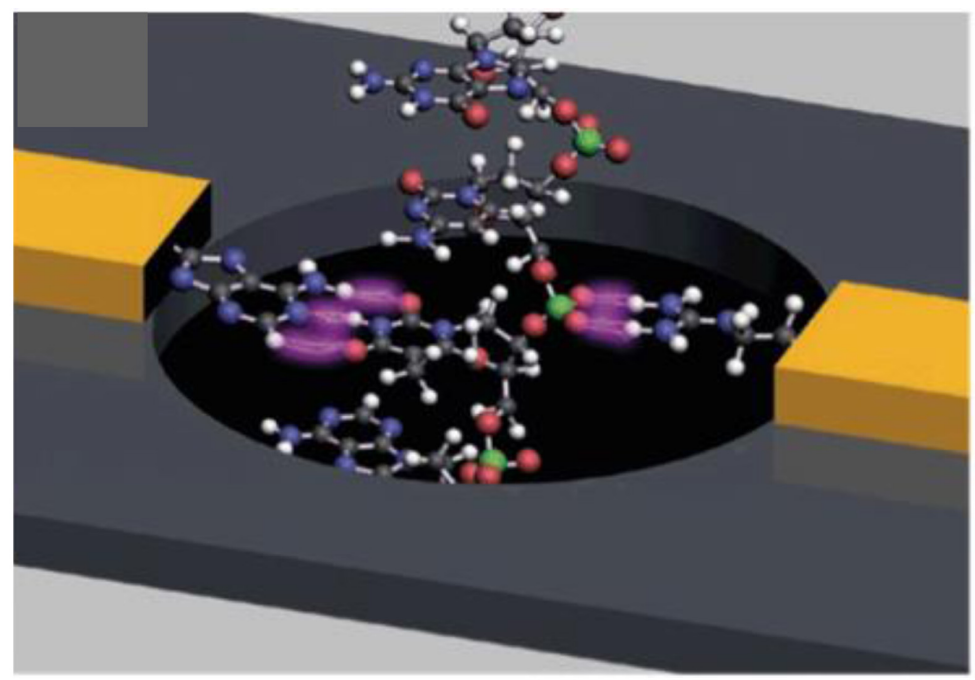

Figure 7. Transverse nanopore electrodes for tunneling current measurements of the DNA backbone with a nanopore. Picture adapted from [100].

To reduce electrical noise, electrodes are covered with a thin $\mathrm{SiO}_{2}$ film. This nanostructure detects molecules passing through the nanopore with the changes in the electric current flowing between the nanogap electrodes, not with the changes in the ionic current flowing parallel to the nanopore. This current between the nanoelectrodes is sourced from a tunneling current conducted via molecules passing through the membrane. Four bases of DNA are distinguishable by their different electrical resistances.

Note that understanding the adsorption properties of DNA bases on electrode (metal) surfaces is fundamental for the rational control of surface functionalization leading to the realization of biocompatible devices for biosensing applications. Investigations of molecule-metal surface interactions that govern the structural and electronic properties of the molecular nanostructures formed upon adsorption are relevant, not only to control surface functionalization for biosensor applications [112] [113] [114] [115], but also to understand the complex biomolecule-surface interactions in general [112] [116].

Tunnel current extremely sensitive to thermal fluctuation in aqueous solution composition and molecule sizes.

Benefits of tunneling readout are:

- direct reads of epigenetic marking, which can be carried out by direct measurement of the pulses of tunnel currents;

- no consumables-purely physical reads;

- simple control of the speed of the molecule, which can be done by selecting the potential of the reference electrode (gate), taking into account the other forces that determine the movement of the molecule in the electrolyte (gravity and drag force).

\subsection{Capacitance Variation Method}

A new design of the sensor forms a flat-plate capacitor with the locally sus- 
pended monolayer MoS2 nanoribbon that functionalized by cytosine molecules, which are complementary to guanine was proposed in [39]. In such a setup the subject to measurement is the modification of capacitance caused by the temporary deflection of the nanoribbon.

The change in capacitance in response to deviations of the tape and the resulting electrical signal is measured using existing integrated circuits without using microscopy techniques. An aqueous functionalized nano-containing $\mathrm{MoS}_{2}$ suspended on a solid electrode as a capacitive displacement sensor is designed to determine the DNA sequence proposed in [39]. The observed sequencing events arise from the combination of the basic Watson-Crick pairing, one of the most basic mechanisms of binding to the key and the key of nature, with the ability of atomically thin membranes of the appropriate size to flex essentially in response to subnanoatomic forces.

DNA sequencing can be done also by the capacitance variation method using ion-sensitive field-effect transistors (ISFET), electrolyte-insulator-semiconductor (EIS) based bio-chemical sensors [117]-[122], and light-addressable potentiometric sensors (LAPS) [123] (Figure 8). In the case of ISFET the threshold voltage changes vs. $\mathrm{pH}$ concentration in bio-liquid or drain current changes vs. gate voltage, for the case EIS the capacitance changes vs. bias voltage, and for the case of LAPS the photocurrent changes vs. bias voltage.

The ISFET (Figure 8(a)) is one of the most popular electrical biosensors. Much attention has been paid to silicon-based biosensors in the field of bio-analytical applications due to their favorable characteristics, which include sensitivity, greater SNR, speed, miniaturization, and low cost. The ISFET, conventionally referred to as a $\mathrm{pH}$ sensor, has been used to measure $\mathrm{H}^{+}$or $\mathrm{OH}^{-}$ions concentration in an aqueous solution, causing an interface potential on the gate insulator $\left(\mathrm{SiO}_{2}\right.$ layer in Figure 8). Many different biosensors have been developed based on $\mathrm{pH}$ sensors since various biomolecular interactions produce protons. The operating principle of the ISFET is simple. Specific molecules are selectively taken into a probe layer on the FET channel, which detects the molecular

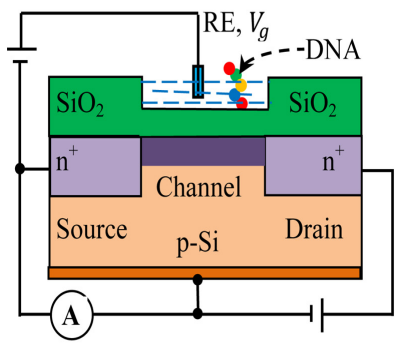

(a)

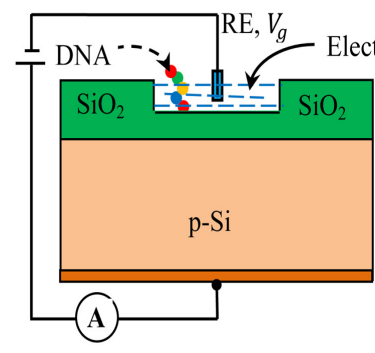

(b)

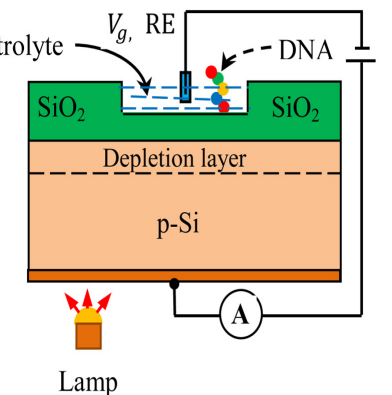

(c)

Figure 8. Schematic illustration of the nanosized bio-chemical sensor structures: (a) ISFET-ion sensitive field effect transistor; (b) EIS-electrolyte-insulator-semiconductor; (c) LAPS-light addressable potentiometric sensor. RE-reference electrode; $V_{g}$ is the gate voltage. 
charge in the probe layer. This charge accumulation shifts the flat band voltage of the field-effect transistor. Effect conditioned with channel conductivity modulation. In the case of DNA detection, the probe is ssDNA with a known sequence, immobilized on the substrate. Since the DNA backbone has intrinsic negative charge, the change in surface charge conditions lead to a change in the FET channel conductance, which can be measured using electrochemical cell consisting of a FET gate as the working electrode together with a reference electrode immersed into a solution. In the case of an ISFET biosensor, the amount of the current flow will be determined by the charges of biomolecules interacting on the gate dielectric. An attractive feature of such FETs is that it is possible to detect biomolecular interactions in a label-free manner through a direct change in conductance or a related electrical property.

A related approach utilizes the changes in the conductance of the semiconductor material, which is attached to the nanopore, when DNA molecules translocate through the channel. This method based on the ISFET biosensors made on nanotubes, nanowires and nanoribons [104] [124]. Those sensors have not long life time because non stability and non-reliability of thin insulator layer (usually $\mathrm{SiO}_{2}, \mathrm{Si}_{3} \mathrm{~N}_{4}$ or $\mathrm{Ta}_{2} \mathrm{O}_{5}$ ) covered semiconductor material (usually silicon) in aqueous solution. Biological or chemical molecules, adsorbed on the surface of the current channel, create a strong chemical bonding.

The EIS capacitor (Figure 8(b)) is one of these modified designs and has been broadly applied for bio-sensing. The EIS structure is identical to that of a metal-oxide-semiconductor (MOS) capacitor but the gate electrode is replaced by an electrolyte medium and a reference electrode. The insulator, commonly an oxide, is thus directly exposed to the electrolyte so changes in the aqueous solution can affect the oxide surface potential and modulate the response of the device.

Estrela et al. employed MOS capacitors consisting of $\mathrm{Au} / \mathrm{SiO}_{2} / \mathrm{Si}$ and poly-Si thin film transistors with a gold metal gate as ISFET biosensor for label-free electrical detection of DNA hybridization [125]. When probe DNA bind to its complementary DNA, changes in electric potential in the electric double layer occur, leading to a shift in the $\mathrm{C}-\mathrm{V}$ (capacitance-voltage) or I-V (current-voltage) characteristics.

When the target ssDNA is supplied, hybridization occurs if the target DNA is complementary to the probe DNA. With or without specific hybridization can be detected by the difference in charge since a nucleotide has a negative charge on the phosphate group.

In the [122] summarizes the FET-based DNA sensors strategies.

The LAPS (Figure $8(\mathrm{c})$ ) is a kind of the ISFET, which has an EIS structure. By illuminating parts of the sensor surface with infrared light, a photocurrent flow of which the amplitude depends on the local surface potential or local $\mathrm{pH}$ value. In this way, the surface potential distribution can be obtained by scanning the light pointer across the surface of LAPS. 


\subsection{Electric Potential Change}

The detection of electric potential change based on an ISFET [126] has shown excellent sensitivity for ion concentration [127], penicillin [128], glucose [129], urease [130], neuronal activity [131], extracellular recording [132], and specific DNA sequence including single nucleotide polymorphisms [1] [87] [133], and so on.

Basic mechanisms of potential generation for electrochemical sensors are [134]:

- $\mathrm{pH}$ or ion-concentration change,

- enzymatic reactions,

- affinity binding of molecules (antigen-antibody affinity reaction, or DNA hybridization),

- potential changes that are coming from living biological systems as a result of more sophisticated biochemical processes (action potential of nerve cells, dipole potentials, etc.).

Detail review of advances and developments in the bio-analytical use of ISFET-based biosensors presented in [118] [135]. An electrochemical sensor for detection of unlabeled ssDNA using peptide nucleic acid (PNA) probes coupled to the FET gate is demonstrated in [136]. An application of ISFET technology for the detection of single nucleotide polymorphisms suggested in [137]. In this study authors developed a useful procedure for sequencing one base via the detection of single-base mismatch in DNA. When DNA strands bind to the gate surface of ISFETs, changes in surface potential of semiconductor occur due to the negative charge of DNA, thereby allowing excellent performance of in DNA sensing. Therefore, the capacitance of the system electrolyte-insulator-semiconductor depletion layer will be changed. Then conductivity of the semiconductor depletion layer will be modulated, and source-drain signal current will be changed. For identification and quantification of individual analytes antibodies are the most commonly used capture agents. Note that for biomolecular recognition in ISFET based sensors it is necessary that biological sensing should take place within the Debye screening length $\lambda_{D}$. In electrolytes, the Debye length is a measure of a charge carrier's net electrostatic effect in a solution and how far its electrostatic effect persists. In an electrolyte or a colloidal suspension, the Debye length for a monovalent electrolyte is [138]

$$
\lambda_{D}=\sqrt{\frac{\varepsilon_{0} \varepsilon_{r} k_{B} T}{2 \times 10^{3} e^{2} N_{A} J}} .
$$

Here $\varepsilon_{0}$ is the permittivity of free space, $\varepsilon_{r}$ is the dielectric constant, $k_{B}$ is the Boltzmann constant, $T$ is the absolute temperature, $e$ is the electron charge, $N_{A}$ is the Avogadro number, $J$ is the ionic strength of the electrolyte in molar units.

The binding of a charged biomolecule result depletion or accumulation of carriers in semiconductor surface layer and cause change of electric charges on 
the gate electrode. In FET biosensors the electric field generating from the binding of a charged biomolecule to the gate is analogous to applying a voltage to a gate. Source-drain current of ISFET can be presented as follows:

$$
I_{d s} \propto \mu C \frac{W}{L} V_{G S}^{2},
$$

where $\mu$ is the charge carrier's mobility in the current channel, $W$ and $L$ sizes of the current channel, $V_{G S}$ is the gate voltage, and

$$
C=\frac{\varepsilon_{0} \varepsilon_{i}}{t_{i}}
$$

is the gate capacitance per unit area, $t_{i}$ is the thickness of the insulator layer; $\varepsilon_{i}$ is the dielectric constant of insulator, respectively.

Note that the sequencing mechanisms of sensors based on ISFETs can be used only for detecting nucleic acid using $\mathrm{pH}$ sensitivity and amplifying the useful signal in real time.

ISFET biosensors have the following applications: DNA-based ISFETs, ISFETs for electro-immunological sensing, enzyme based-ISFETs, ISFETs for monitoring living cell responses.

\section{Some Notes (Criticism) and Conclusion}

The challenges for next generation DNA sequencing are to have robust, stable and reproducible nanosized sensor-devices. One major challenge of nanopore-based DNA sequencing technologies is to find an efficient way to reduce DNA translocation speed so that each nucleotide can reside long enough in the pore for reading and forming "long"-time and accurate recognition. The existing methods of DNA sequencing are still not well optimized from the point of view of cost and DNA translocation speed. Detailed analyses show that the main problems of electrical detection methods for nanopore-based DNA sequencing are:

- high translocation speed of molecules through the pore (in bases/s: 1000 [139], 9600 [140], 55,000 [135] [141], 70,000,000 [39]);

- low level of desired electrical signal (of the order of pA for ionic blockade currents [87] [97]-[104] and of the order of nA for tunnel currents [19] [65] [87] [142] [143]);

- low value of the signal-to-noise ratio (for biological and graphene nanopores $<10[40])$

- etc.

In the other hand to achieve high-quality sequencing performance, solid-state nanopores should have the following characteristics:

- the size of the nanopores should be comparable to the DNA molecule diameter ( 1 $\mathrm{nm}$ for ssDNA and $\sim 2 \mathrm{~nm}$ for dsDNA);

- the effective length/thickness of the nanopore should be no more than the distance between two adjacent bases ( $\sim 0.5 \mathrm{~nm}$ for ssDNA) to realize sin- 
gle-base sequencing precision.

Note that all of the biological and synthetic nanopores have barrels of $\sim 5 \mathrm{~nm}$ (which is considerably longer than the base-to-base distance of $3.4 \AA$ ) in thickness and accommodate $\sim 10$ - 15 nucleotides at a time. It is, therefore, impossible to achieve single-base resolution using blockage current measurements. In addition, the average rate at which a polymer typically translocates through a nanopore is on the order of 1 nucleotide/ $\mu$ s (i.e., on the order of $\mathrm{MHz}$ detection), which is too fast to resolve. The time distribution of two processes (capture, entry, and translocation) is non-Poisson and often differs by an order of magnitude. This means that two molecules pass through a nanopore at considerably different rates and the slower one could be missed or misinterpreted.

Analysis conducted above revealed the following.

1) Case of Ionic Current Blockade

It is well known that ionic current $I$ is carried out by mass transfer

$$
m=k I t_{0},
$$

where $k$ is the electrochemical equivalent, $t_{0}$ is the current passing time (see Figure 2). By connecting electrons from the cathode, the protons in aqueous solution are converted into hydrogen atoms, which are converted into a hydrogen molecule. The latter must be removed from the electrolyte near the cathode. The movement of hydrogen molecules will create some fluctuation around the cathode in the aqueous solution. As a result, the solution homogeneity will be disturbed, which will lead to increased current noise and decreased signal-to-noise ratio. Other ions which are in solution can come closer the corresponding electrodes take or give away electrons and turn into neutral atoms. Some of these atoms can be deposited on the electrodes, some at the bottom of the chamber. The process of atoms deposition on the electrodes is the random process and will repeat many times when pore is empty. Such process will inevitably lead to a reduction in pore size. Then pore will be closed for the following DNA molecules. To restore the sensor, it will be necessary to open a new pore. This means that the re-productivity of the DNA sensing and reading process will be very low. On the other hand, the ionic current has a very low value (of the order of picoamperes), and therefore it will be very sensitive to any fluctuations (solution homogeneity, pore diameter, etc.). So, the noise level will be high, and the SNR value will be low.

\section{2) Case of Tunnel Current}

Note following. In the case of the single layer graphene nanopore sequencing effective thickness (the covalent diameter of carbon) is equal to $0.15 \mathrm{~nm}$ [144] and for the effective sequencing will be used at least 3 - 5 layered graphene nanoribbon. Let us turn more detail to the case shown in Figure 4 (adapted from [49]), which is very common in the literature (see also [52]). There presented usage of graphene nanoribbons for transverse current modulation by DNA in two-terminal devices. In [49] we read: “Unlike other recent biosensors based on transverse electronic transport through translocated DNA, which utilize small 
(of the order of $p A$ ) tunneling current across a nanogap or a nanopore yielding a poor signal-to-noise ratio, our device concept relies on the fact that in ZGNRs (zigzag nanoribbon) local current density is peaked around the edges so that drilling a nanopore away from the edges will not diminish the conductance. Inserting a nucleobase into the nanopore affects the charge density in the surrounding area, thereby modulating edge conduction currents ...". In [52] we read: "The nucleobases were inserted into a pore in a graphene nanoribbon, and the electrical current and conductance spectra were calculated as function of voltage applied across the nanoribbon."

In this regard, it is necessary to note the following.

The total current between the electrodes is equal to the sum of the conduction current of the continuous layer near the pores and the tunneling current through nucleobase (compare Figure 4 and Figure 9). It is known that the conductivity of graphene in continuous regions is much greater than the conductivity of the nucleobases of the DNA molecule in the pore. Note that at room temperature, graphene has slightly better conductivity than silver. Electron and hole mobilities of up to $200,000 \mathrm{~cm}^{2} / \mathrm{V} \cdot \mathrm{s}$ and high maximum current densities of $\sim 2 \mathrm{~mA} / \mu \mathrm{m}$ in width, or $6 \times 10^{9} \mathrm{~A} / \mathrm{cm}^{2}$ have been measured in graphene [145]. On the other hand, the conductivity of organic molecules (including nucleobases) is much smaller (electrical resistances are usually on the order of Mom) and they are closer to dielectrics than conductors. With such parameters, the conduction current through a continuous layer of graphene will be much greater than the tunneling current. This means that the measured current, both in the absence and in the presence of DNA in the pore, will be exactly the conduction current and its fluctuation will be very insignificant.

At the transverse nanopore electrodes presented in Figure 7 the capture cross section area of the electrodes for the DNA molecule is very small and the conductive bridge can be created only with the appropriate orientation of the molecule. The probability of the molecule capture will be low.

Note also that comparing with the ionic blockade current level of the tunnel

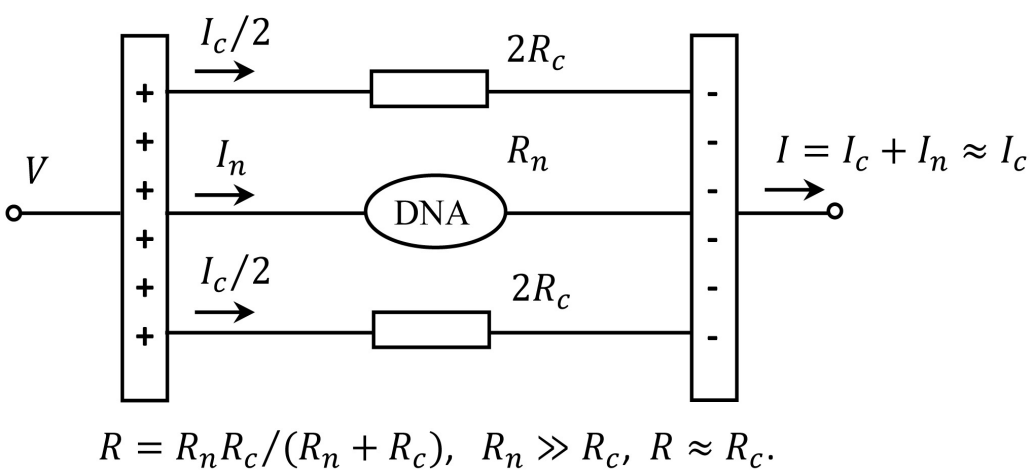

Figure 9. Equivalent scheme of two-terminal device for transverse current modulation made on graphene nanoribbons (see Ref. [49]). $R_{n}$ and $R_{c}$ are resistances of nucleobase and continuous region of graphene, $I_{c}, I_{n}$ are currents through continuous region and nucleobase, $I$ is the total current. 
current is higher and it is not more sensitive to any fluctuations (for example solution homogeneity).

3) Case of the change in capacitance

For the case of a design of the sensor forms a flat-plate capacitor with the locally suspended monolayer MoS2 nanoribbon proposed in [39] where the subject to measurement is the modification of capacitance caused by the temporary deflection of the nanoribbon note following. The change in capacitance in response to deviations of the tape and the measured resulting electrical signal must be very low and therefore they will be hard measurable and very sensitive to any fluctuations. Note that average deflection was $\sim(0.1-0.2) \AA$ or $(0.01-0.02) \mathrm{nm}$ (Figure 2 [39]).

\section{4) Case ISFET and EIS sensors}

Sequencing mechanisms of sensors based on ISFETs can actually be used only for detecting nucleic acid using $\mathrm{pH}$ sensitivity and amplifying the useful signal in real time. For the ISFET and capacitive EIS biosensors we can note:

- non-stability and non-reliability of work because of thin insulator layer (6 - 8 $\mathrm{nm})$,

- random character of the capture of molecules on the sensing layer,

- etc.

To realize more correct, precise and more measurable (comparatively high currents), low noisy DNA sequencing it is necessary to use new, more optimal architecture of nanopore and as an information signal use tunnel current through metal-nucleotide-metal junctions. One variant of the optimal architecture developed by us presented in [146].

\section{Conflicts of Interest}

The authors declare no conflicts of interest regarding the publication of this paper.

\section{References}

[1] Watson, J.D. and. Crick F.H.C. (1953) Molecular Structure of Nucleic Acids: A Structure for Deoxyribose Nucleic Acid. Nature, 171, 737-738. https://doi.org/10.1038/171737a0

[2] Service, R.F. (2006) The Race for the $\$ 1000$ Genome. Science, 311, 1544-1546. https://doi.org/10.1126/science.311.5767.1544

[3] Fanget, A. (2013) Towards Tunneling Electrodes for Nanopore-Based DNA Sequencing. Thèse No 5700. Ecole Polytechnique Fèdèrale de Lausanne, Suisse.

[4] Raza, S. and Ameen, A. (2017) Nano Pore Sequencing Technology: A Review. International Journal of Advances in Scientific Research, 3, 90-95. https://doi.org/10.7439/ijasr.v3i8.4333

[5] Pillai, S., Gopalan, V. and Lam, A.K.-Y. (2017) Review of Sequencing Platforms and Their Applications in Phaeochromocytoma and Paragangliomas. Critical Reviews in Oncologyl Hematology, 116, 58-67.

[6] Ansorge, W.J. (2016) Next Generation DNA Sequencing (II): Techniques, Applica- 
tions. Journal of Next Generation Sequencing and Applications, No. S1, 5.

[7] Sanger, F. and Coulson, A.R. (1975) A Rapid Method for Determining Sequences in DNA by Primed Synthesis with DNA Polymerase. Journal of Molecular Biology, 94, 441-446. https://doi.org/10.1016/0022-2836(75)90213-2

[8] Sanger, F., Nicklen, S. and Coulson, A.R. (1977) DNA Sequencing with Chain-Terminating Inhibitors. Proceedings of the National Academy of Sciences of the United States of America, 74, 5463-5467. https://doi.org/10.1073/pnas.74.12.5463

[9] Maxam, A.M. and Gilbert, W. (1977) A New Method for Sequencing DNA. Proceedings of the National Academy of Sciences of the United States of America, 74, 560-564. https://doi.org/10.1073/pnas.74.2.560

[10] Anderson, S. (1981) Article Navigation Shotgun DNA Sequencing Using Cloned DNase I-Generated Fragments. Nucleic Acids Research, 9, 3015-3027. https://doi.org/10.1093/nar/9.13.3015

[11] Canard, B. and Sarfati, R.S. (1994) DNA Polymerase Fluorescent Substrates with Reversible 3'-Tags. Gene, 148, 1-6. https://doi.org/10.1016/0378-1119(94)90226-7

[12] Nyren, P., Petersson, B. and Uhlen, M. (1993) Solid Phase DNA Minisequencing by an Enzymatic Luminometric Inorganic Pyrophosphate Detection Assay. Analytical Biochemistry, 208, 171-175.

[13] Hultman, S., Stahl, S., Homes, E. and Uhlén, M. (1989) Direct Solid Phase Sequencing of Genomic and Plasmid DNA Using Magnetic Beads as Solid Support. Nucleic Acids Research, 17, 4937-4946. https://doi.org/10.1093/nar/17.13.4937

[14] Loman, N.J., Quick, J. and Simpson, J.T. (2015) A Complete Bacterial Genome Assembled de Novo Using Only Nanopore Sequencing Data. Nature Methods, 12, 733-735. https://doi.org/10.1038/nmeth.3444

[15] Lin, X., Ivanov, A.P. and Edel, J.B. (2017) Selective Single Molecule Nanopore Sensing of Proteins Using DNA Aptamer-Functionalised Gold Nanoparticles. Chem. Sci., 8, 3905-3912. https://doi.org/10.1039/C7SC00415J

[16] Wloka, C., Van Meervelt, V., Van Gelder, D., Danda, N., Jager, N., Williams, C.P. and Maglia, G. (2017) Label-Free and Real-Time Detection of Protein Ubiquitination with a Biological Nanopore. ACS Nano, 11, 4387-4394.

https://doi.org/10.1021/acsnano.6b07760

[17] Kennedy, E., Dong, Z., Tennant, C. and Timp, G. (2016) Reading the Primary Structure of a Protein with $0.07 \mathrm{~nm}^{3}$ Resolution Using a Subnanometre-Diameter Pore. Nature Nanotechnology, 11, 968-976.

https://doi.org/10.1038/nnano.2016.120

[18] Plesa, C., Ruitenberg, J.W., Witteveen, M.J. and Dekker, C. (2015) Detection of Individual Proteins Bound along DNA Using Solid-State Nanopores. Nano Letters, 15, 3153-3158. https://doi.org/10.1021/acs.nanolett.5b00249

[19] Clarke, J., Wu, H.-C., Jayasinghe, L., Patel, A., Reid, S. and Bayley, H. (2009) Continuous Base Identification for Single-Molecule Nanopore DNA Sequencing. Nature Nanotechnology, 4, 265-270. https://doi.org/10.1038/nnano.2009.12

[20] Farimani, A.B., Heiranian, M., Min, K. and Aluru, N.R. (2017) Antibody Subclass Detection Using Graphene Nanopores. The Journal of Physical Chemistry Letters, 8, 1670-1676. https://doi.org/10.1021/acs.jpclett.7b00385

[21] Wang, S., Haque, F., Rychahou, P.G., Evers, B.M. and Guo, P. (2013) Engineered Nanopore of Phi29 DNA-Packaging Motor for Real-Time Detection of Single Colon Cancer Specific Antibody in Serum. ACS Nano, 7, 9814-9822. 
https://doi.org/10.1021/nn404435v

[22] Shi, J., Hou, J. and Fang, Y. (2016) Recent Advances in Nanopore-Based Nucleic Acid Analysis and Sequencing. Microchimica Acta, 183, 925-939.

https://doi.org/10.1007/s00604-015-1503-y

[23] Ying, Y.-L., Zhang, J., Gao, R. and Long, Y.-T. (2013) Nanopore-Based Sequencing and Detection of Nucleic Acids. Angewandte Chemie International Edition, 52, 13154-13161. https://doi.org/10.1002/anie.201303529

[24] Rincon-Restrepo, M., Mikhailova, E., Bayley, H. and Maglia, G. (2011) Controlled Translocation of Individual DNA Molecules through Protein Nanopores with Engineered Molecular Brakes. Nano Letters, 11, 746-750.

https://doi.org/10.1021/nl1038874

[25] Howorka, S., Cheley, S. and Bayley, H. (2001) Sequence-Specific Detection of Individual DNA Strands Using Engineered Nanopores. Nature Biotechnology, 19, 636-639. https://doi.org/10.1038/90236

[26] Zahid, O.K., Wang, F., Ruzicka, J.A., Taylor, E.W. and Hall, A.R. (2016) Sequence-Specific Recognition of MicroRNAs and Other Short Nucleic Acids with Solid-State Nanopores. Nano Letters, 16, 2033-2039.

https://doi.org/10.1021/acs.nanolett.6b00001

[27] Clamer, M., Höfler, L., Mikhailova, E., Viero, G. and Bayley, H. (2014) Detection of 3'-End RNA Uridylation with a Protein Nanopore. ACS Nano, 8, 1364-1374. https://doi.org/10.1021/nn4050479

[28] Bulushev, R.D., Marion, S. and Radenovic, A. (2015) Relevance of the Drag Force during Controlled Translocation of a DNA-Protein Complex through a Glass $\mathrm{Na}$ nocapillary. Nano Letters, 15, 7118-7125.

https://doi.org/10.1021/acs.nanolett.5b03264

[29] He, H., Xu, X., Wang, P., Chen, L. and Jin, Y. (2015) The Facile Surface Chemical Modification of a Single Glass Nanopore and Its Use in the Nonenzymatic Detection of Uric Acid. Chemical Communications, 51, 1914-1917. https://doi.org/10.1039/C4CC09185I

[30] Yin, B., Xie, W., Liang, L., Deng, Y., He, S., He, F., Zhou, D., Tlili, C. and Wang, D. (2017) Covalent Modification of Silicon Nitride Nanopore by Amphoteric Polylysine for Short DNA Detection. ACS Omega, 2, 7127-7135.

https://doi.org/10.1021/acsomega.7b01245

[31] Shekar, S., Niedzwiecki, D.J., Chien, C.C., Ong, P., Fleischer, D.A., Lin, J., Rosenstein, J.K., Drndic, M. and Shepard, K.L. (2016) Measurement of DNA Translocation Dynamics in a Solid-State Nanopore at $100 \mathrm{~ns}$ Temporal Resolution. Nano Letters, 16, 4483-4489. https://doi.org/10.1021/acs.nanolett.6b01661

[32] Liang, Z., Tang, Z., Li, J., Hu, R., Yu, D. and Zhao, Q. (2015) Interaction Prolonged DNA Translocation through Solid-State Nanopores. Nanoscale, 7, 10752-10759. https://doi.org/10.1039/C5NR01954K

[33] Montagne, F., Blondiaux, N., Bojko, A. and Pugin, R. (2012) Molecular Transport through Nanoporous Silicon Nitride Membranes Produced from Self-Assembling Block Copolymers. Nanoscale, 4, 5880-5886. https://doi.org/10.1039/c2nr31498c

[34] Venkatesan, B.M., Shah, A.B., Zuo, J.-M. and Bashir, R. (2010) DNA Sensing Using Nanocrystalline Surface-Enhanced $\mathrm{Al}_{2} \mathrm{O}_{3}$ Nanopore Sensors. Advanced Functional Materials, 20, 1266-1275. https://doi.org/10.1002/adfm.200902128

[35] Zwolak, M. and Di Ventra, M. (2005) Electronic Signature of DNA Nucleotides via Transverse Transport. Nano Letters, 5, 421-424. 
https://doi.org/10.1021/nl048289w

[36] Yin, K., Huang, S., Chen, X., Wang, X., Kong, J., Chen, Y. and Xue, J. (2018) Generating Sub-Nanometer Pores in Single-Layer $\mathrm{MoS}_{2}$ by Heavy-Ion Bombardment for Gas Separation: A Theoretical Perspective. ACS Applied Materials \& Interfaces, 10, 28909-28917. https://doi.org/10.1021/acsami.8b10569

[37] Luan, B. and Zhou, R. (2018) Single-File Protein Translocations through Graphene- $\mathrm{MoS}_{2}$ Heterostructure Nanopores. The Journal of Physical Chemistry Letters, 9, 3409-3415. https://doi.org/10.1021/acs.jpclett.8b01340

[38] Luan, B. and Zhou, R. (2018) Spontaneous Transport of Single-Stranded DNA through Graphene- $\mathrm{MoS}_{2}$ Heterostructure Nanopores. ACS Nano, 12, 3886-3891. https://doi.org/10.1021/acsnano.8b01297

[39] Smolyanitsky, A., Yakobson, B.I., Wassenaar, T.A., Paulechka, E. and Kroenlein, K. (2016) $\mathrm{A} \mathrm{MoS}_{2}$-Based Capacitive Displacement Sensor for DNA Sequencing. ACS Nano, 10, 9009-9016. https://doi.org/10.1021/acsnano.6b05274

[40] Farimani, A.B., Min, K. and Aluru, N.R. (2014) DNA Base Detection Using a Single-Layer $\mathrm{MoS}_{2}$. ACS Nano, 8, 7914-7922. https://doi.org/10.1021/nn5029295

[41] De Souza, F.A.L., Amorim, R.G., Scopel, W.L. and Scheicher, R.H. (2017) Electrical Detection of Nucleotides via Nanopores in a Hybrid Graphene/h-BN Sheet. Nanoscale, 9, 2207-2212. https://doi.org/10.1039/C6NR07154F

[42] Gu, Z., Zhang, Y., Luan, B. and Zhou, R. (2016) DNA Translocation through Single-Layer Boron Nitride Nanopores. Soft Matter, 12, 817-823.

https://doi.org/10.1039/C5SM02197A

[43] Zhang, L. and Wang, X. (2016) DNA Sequencing by Hexagonal Boron Nitride Nanopore: A Computational Study. Nanomaterials, 6, 111-121. https://doi.org/10.3390/nano6060111

[44] Shukla, V., Jena, N.K., Grigoriev, A. and Ahuja, R. (2017) Prospects of Graphene-hBN Heterostructure Nanogap for DNA Sequencing. ACS Applied Materials \& Interfaces, 9, 39945-39952. https://doi.org/10.1021/acsami.7b06827

[45] Amorim, R.G., Rocha, A.R. and Scheicher, R.H. (2016) Boosting DNA Recognition Sensitivity of Graphene Nanogaps through Nitrogen Edge Functionalization. Journal of Physical Chemistry C, 120, 19384-19388.

https://doi.org/10.1021/acs.jpcc.6b04683

[46] Prasongkit, J., Grigoriev, A., Pathak, B., Ahuja, R. and Scheicher, R.H. (2013) Theoretical Study of Electronic Transport through DNA Nucleotides in a Double-Functionalized Graphene Nanogap. Journal of Physical Chemistry C, 117, 15421-15428. https://doi.org/10.1021/jp4048743

[47] Takeuchi, K. and Zolotoukhina, T. (2013) Individual DNA Base Identification at the Transport through Graphene Nanopore. ASME 11th International Conference on Nanochannels, Microchannels, and Minichannels, Sapporo, Japan, 16-19 June 2013, V001T10A003.

[48] Traversi, F., Raillon, C., Benameur, S.M., et al. (2013) Detecting the Translocation of DNA through a Nanopore Using Graphene Nanoribbons. Nature Nanotechnology, 8, 939-945. https://doi.org/10.1038/nnano.2013.240

[49] Saha, K.K., Drndić, M. and Nikolić, B.K. (2012) DNA Base-Specific Modulation of Microampere Transverse Edge Currents through a Metallic Graphene Nanoribbon with a Nanopore. Nano Letters, 12, 50-55. https://doi.org/10.1021/nl202870y

[50] Merchant, C.A., Healy, K., Wanunu, M., Ray, V., Peterman, N., Bartel, J., Fischbein, M.D., Venta, K., Luo, Z., Johnson, A.T.C. and Drndić, M. (2010) DNA Transloca- 
tion through Graphene Nanopores. Nano Letters, 10, 2915-2921. https://doi.org/10.1021/nl101046t

[51] Schneider, G.F., Kowalczyk, S.W., Calado, V.E., Pandraud, G., Zandbergen, H.W., Vandersypen, L.M.K. and Dekker, C. (2010) DNA Translocation through Graphene Nanopores. Nano Letters, 10, 3163-3167. https://doi.org/10.1021/nl102069z

[52] Nelson, T., Zhang, B. and Prezhdo, O.V. (2010) Detection of Nucleic Acids with Graphene Nanopores: Ab Initio Characterization of a Novel Sequencing Device. Nano Letters, 10, 3237-3242. https://doi.org/10.1021/nl9035934

[53] Yang, N. and Jiang, X. (2017) Nanocarbons for DNA Sequencing: A Review. Carbon, 115, 293-311. https://doi.org/10.1016/j.carbon.2017.01.012

[54] Liu, L., Xie, J., Li, T. and Wu, H.-C. (2015) Fabrication of Nanopores with Ultrashort Single-Walled Carbon Nanotubes Inserted in a Lipid Bilayer. Nature Protocols, 10, 1670-1678. https://doi.org/10.1038/nprot.2015.112

[55] Zhang, S., Wang, X., Li, T., Liu, L., Wu, H.C., Luo, M. and Li, J. (2015) Sensitive Detection of a Modified Base in Single-Stranded DNA by a Single-Walled Carbon Nanotube. Langmuir, 31, 10094-10099. https://doi.org/10.1021/acs.langmuir.5b01272

[56] Kim, H.S., Lee, S.J. and Kim, Y.-H. (2014) Distinct Mechanisms of DNA Sensing Based on N - Doped Carbon Nanotubes with Enhanced Conductance and Chemical Selectivity. Small, 10, 774-781. https://doi.org/10.1002/smll.201301225

[57] Liu, L., Yang, C., Zhao, K., Li, J. and Wu, H.C. (2013) Ultrashort Single-Walled Carbon Nanotubes in a Lipid Bilayer as a New Nanopore Sensor. Nature Communications, 4, Article No. 2989. https://doi.org/10.1038/ncomms3989

[58] Göpfrich, K., Li, C.-Y., Mames, I., Bhamidimarri, S.P., Ricci, M., Yoo, J., Mames, A., Ohmann, A., Winterhalter, M., Stulz, E., Aksimentiev, A. and Keyser, U.F. (2016) Ion Channels Made from a Single Membrane-Spanning DNA Duplex. Nano Letters, 16, 4665-4669. https://doi.org/10.1021/acs.nanolett.6b02039

[59] Di Ventra, M. and Taniguchi, M. (2016) Decoding DNA, RNA and Peptides with Quantum Tunneling. Nature Nanotechnology, 11, 117-126. https://doi.org/10.1038/nnano.2015.320

[60] Gao, H.L., Wang, M., Wu, Z.Q., Wang, C., Wang, K. and Xia, X.H. (2015) Morpholino-Functionalized Nanochannel Array for Label-Free Single Nucleotide Polymorphisms Detection. Analytical Chemistry, 87, 3936-3941. https://doi.org/10.1021/ac504830e

[61] Agah, S., Zheng, M., Pasquali, M. and Kolomeisky, A.B. (2016) DNA Sequencing by Nanopores: Advances and Challenges. Journal of Physics D: Applied Physics, 49, Article ID: 413001. https://doi.org/10.1088/0022-3727/49/41/413001

[62] Laszlo, A., Derrington, I., Ross, B., Brinkerhoff, H., Adey, A., Nova, I., Craig, J., Langford, K., Samson, J.M., Daza, R., Doering, K., Shendure, J. and Gundlach, J. (2014) Decoding Long Nanopore Sequencing Reads of Natural DNA. Nature Biotechnology, 32, 829-833. https://doi.org/10.1038/nbt.2950

[63] Venkatesan, B.M. and Bashir, R. (2011) Nanopore Sensors for Nucleic Acid Analysis. Nature Nanotechnology, 6, 615-624. https://doi.org/10.1038/nnano.2011.129

[64] Panja, D., Barkema, G.T. and Kolomeisky, A.B. (2013) Through the Eye of the Needle: Recent Advances in Understanding Biopolymer Translocation. Journal of Physics: Condensed Matter, 25, Article ID: 413101. https://doi.org/10.1088/0953-8984/25/41/413101

[65] Meller, A. (2003) Dynamics of Polynucleotide Transport through Nanometre-Scale 
Pores. Journal of Physics. Condensed Matter, 15, R581-R607.

[66] Liu, Z., Wang, Y., Deng, T. and Chen, Q. (2016) Solid-State Nanopore-Based DNA Sequencing Technology. Journal of Nanomaterials, 2016, Article ID: 5284786. https://doi.org/10.1155/2016/5284786

[67] Timp, W., Mirsaidov, U.M., Wang, D., Comer, J., Aksimentiev, A. and Timp, G. (2010) Nanopore Sequencing: Electrical Measurements of the Code of Life. IEEE Transactions on Nanotechnology, 9, 281-294. https://doi.org/10.1109/TNANO.2010.2044418

[68] Shi, W., Friedman, A.K. and Baker, L.A. (2017) Nanopore Sensing. Analytical Chemistry, 89, 157-188. https://doi.org/10.1021/acs.analchem.6b04260

[69] Garrido-Cardenas, J., Garcia-Maroto, F., Alvarez-Bermejo, J. and Manzano-Agugliaro, F. (2017) DNA Sequencing Sensors: An Overview. Sensors, 17, 588-602. https://doi.org/10.3390/s17030588

[70] Liu, L. and Wu, H.C. (2016) DNA-Based Nanopore Sensing. Angewandte Chemie International Edition, 55, 15216-15222. https://doi.org/10.1002/anie.201604405

[71] Deamer, D., Akeson, M. and Branton, D. (2016) Three Decades of Nanopore Sequencing. Nature Biotechnology, 34, 518-524. https://doi.org/10.1038/nbt.3423

[72] Goodwin, S., McPherson, J.D. and McCombie, W.R. (2016) Coming of Age: Ten Years of Next-Generation Sequencing Technologies. Nature Reviews Genetics, 17, 333-351. https://doi.org/10.1038/nrg.2016.49

[73] Marx, V. (2015) Nanopores: A Sequencer in Your Backpack. Nature Methods, 12, 1015-1018. https://doi.org/10.1038/nmeth.3625

[74] Shendure, J.A., Porreca, G.J., Church, G.M., Gardner, A.F., Hendrickson, C., Kieleczawa, J. and Slatko, B.E. (2011) Overview of DNA Sequencing Strategies. In: Current Protocols in Molecular Biology, Chapter 7, Unit 7.1., 1-23.

[75] Linnarsson, S. (2010) Recent Advances in DNA Sequencing Methods-General Principles of Sample Preparation. Experimental Cell Research, 316, 1339-1343. https://doi.org/10.1016/j.yexcr.2010.02.036

[76] Carson, S. and Wanunu, M. (2015) Challenges in DNA Motion Control and Sequence Readout Using Nanopore Devices. Nanotechnology, 26, Article ID: 074004.

[77] Lee, K., Lee, H., Lee, S.-H., Kim, H.-M., Kim, K.-B. and Kim, S.J. (2017) Enhancing the Sensitivity of DNA Detection by Structurally Modified Solid-State Nanopore. Nanoscale, 9, 18012-18021. https://doi.org/10.1039/C7NR05840C

[78] Gusakova, J., Wang, X., Shiau, L.L., Krivosheeva, A., Shaposhnikov, V., Borisenko, V., Gusakov, V. and Tay, B.K. (2017) Electronic Properties of Bulk and Monolayer TMDs: Theoretical Study within DFT Framework (GVJ-2e Method). Physica Status Solidi (a), 214, Article ID: 1700218. https://doi.org/10.1002/pssa.201700218

[79] Kneipp, K., Kneipp, H., Kartha, V.B., Manoharan, R., Deinum, G., Itzkan, I., Dasari, R.R. and Feld, M.S. (1998) Detection and Identification of a Single DNA Base Molecule Using Surface-Enhanced Raman Scattering (SERS). Physical Review E, 57, R6281(R). https://doi.org/10.1103/PhysRevE.57.R6281

[80] Afsari, S., Korshoj, L.E., Abel Jr., G.R., Khan, S., Chatterjee, A. and Nagpal, P. (2017) Quantum Point Contact Single-Nucleotide Conductance for DNA and RNA Sequence Identification. ACS Nano, 11, 11169-11181. https://doi.org/10.1021/acsnano.7b05500

[81] Soni, G.V. and Meller, A. (2007) Progress toward Ultrafast DNA Sequencing Using Solid-State Nanopores. Clinical Chemistry, 53, 1996-2001.

https://doi.org/10.1373/clinchem.2007.091231 
[82] United States Food and Drug Administration (2016) Nucleic Acid Based Tests. http://www.fda.gov/MedicalDevices/ProductsandMedicalProcedures/InVitroDiagn ostics/ucm330711.htm

[83] Chen, K., Juhasz, M., Gularek, F., Weinhold, E., Tian, Y., Keyser, U.F. and Bell, N.A.W. (2017) Ionic Current-Based Mapping of Short Sequence Motifs in Single DNA Molecules Using Solid-State Nanopores. Nano Letters, 17, 5199-5205. https://doi.org/10.1021/acs.nanolett.7b01009

[84] Larkin, J., Henley, R., Bell, D.C., Cohen-Karni, T., Rosenstein, J.K. and Wanunu, M. (2013) Slow DNA Transport through Nanopores in Hafnium Oxide Membranes. ACS Nano, 7, 10121-10128. https://doi.org/10.1021/nn404326f

[85] Moretti, M., Di Fabrizio, E., Cabrini, S.R., De Angelis, F. and Firrao, G. (2008) An ON/OFF Biosensor Based on Blockade of Ionic Current Passing through a Solid-State Nanopore. Biosensors and Bioelectronics, 24, 141-147. https://doi.org/10.1016/j.bios.2008.03.047

[86] Shendure, J., Balasubramanian, S., Church, G.M., Gilbert, W., Rogers, J., Schloss, A.J. and Waterston, R.H. (2017) DNA Sequencing at 40: Past, Present and Future. Nature, 550, 345-353. https://www.nature.com/articles/nature24286 https://doi.org/10.1038/nature24286

[87] Butler, T.Z., Gundlach, J.H. and Troll, M. (2007) Ionic Current Blockades from DNA and RNA Molecules in the $\alpha$-Hemolysin Nanopore. Biophysical Journal, 93, 3229-3240. https://doi.org/10.1529/biophysj.107.107003

[88] He, J., Lin, L., Zhang, P. and Lindsay, S. (2007) Identification of DNA Basepairing via Tunnel-Current Decay. Nano Letters, 7, 3854-3858. https://doi.org/10.1021/nl0726205

[89] Zwolak, M. and Di Ventra, M. (2008) Colloquium: Physical Approaches to DNA Sequencing and Detection. Reviews of Modern Physics, 80, 141-165. https://doi.org/10.1103/RevModPhys.80.141

[90] Xu, M., Endres, R.G. and Arakawa, Y. (2007) The Electronic Properties of DNA Bases. Small, 3, 1539-1543. https://doi.org/10.1002/smll.200600732

[91] Taniguchi, M. (2014) Single-Molecule Sequencing Technologies of Biomolecules via Electric Currents. 18th International Conference on Miniaturized Systems for Chemistry and Life Sciences, San Antonio, TX, 26-30 October 2014, 199-204.

[92] Gracheva, M.E., Xiong, A.L., Aksimentiev, A., Schulten, K., Timp, G. and Leburton, J.-P. (2006) Simulation of the Electric Response of DNA Translocation through a Semiconductor Nanopore-Capacitor. Nanotechnology, 17, 622-633. https://doi.org/10.1088/0957-4484/17/3/002

[93] Gracheva, M.E., Aksimentiev, A. and Leburton, J.-P. (2006) Electrical Signatures of Single-Stranded DNA with Single Base Mutations in a Nanopore Capacitor. Nanotechnology, 17, 3160-3165. https://doi.org/10.1088/0957-4484/17/13/014

[94] Heng, J.B., Aksimentiev, A., Ho, C., Dimitrov, V., Sorsch, T., Miner, J., Mansfield, W., Schulten K. and Timp, G. (2005) Beyond the Gene Chip. Bell LabsTechnical Journal, 10, 5-22. https://doi.org/10.1002/bltj.20102

[95] Milgrew, M.J. and Cumming, D.R.S. (2008) Matching the Transconductance Characteristics of CMOS ISFET Arrays by Removing Trapped Charge. IEEE Transactions on Electron Devices, 55, 1074-1079. https://doi.org/10.1109/TED.2008.916680

[96] Maruyama, Y., Terao, S. and Sawada, K. (2009) Label Free CMOS DNA Image Sensor Based on the Charge Transfer Technique. Biosensors and Bioelectronics, 24, 3108-3112. https://doi.org/10.1016/j.bios.2009.03.031 
[97] Kasianowicz, J.J., Brandin, E., Branton, D. and Deamer, D.W. (1996) Characterization of Individual Polynucleotide Molecules Using a Membrane Channel. Proceedings of the National Academy of Sciences of the United States of America, 93, 13770-13773. https://doi.org/10.1073/pnas.93.24.13770

[98] Dekker, C. (2007) Solid-State Nanopores. Nature Nanotechnology, 2, 209-215. https://doi.org/10.1038/nnano.2007.27

[99] Deamer, D.W. and Branton, D. (2002) Characterization of Nucleic Acids by Nanopore Analysis. Accounts of Chemical Research, 35, 817-825. https://doi.org/10.1021/ar000138m

[100] Branton, D., Deamer, D.W., Marziali, A., Bayley, H., Benner, S.A., Butler, T., Di Ventra, M., Garaj, S., Hibbs, A., Huang, X., Jovanovich, S.B., Krstic, P.S., Lindsay, S., Ling, X.S., Mastrangelo, C.H., Meller, A., Oliver, J.S., Pershin, Y.V., Ramsey, J.M., Riehn, R., Soni, G.V., Tabard-Cossa, V., Wanunu, M., Wiggin, M. and Schloss, J.A. (2008) The Potential and Challenges of Nanopore Sequencing. Nature Biotechnology, 26, 1146-1153. https://doi.org/10.1038/nbt.1495

[101] Bayley, H. (2006) Sequencing Single Molecules of DNA. Current Opinion in Chemical Biology, 10, 628-637. https://doi.org/10.1016/j.cbpa.2006.10.040

[102] Ashkenasy, N., Sanchez-Quesada, J., Bayley, H. and Ghadiri, M.R. (2005) Recognizing a Single Base in an Individual DNA Strand: A Step Toward DNA Sequencing in Nanopores. Angewandte Chemie International Edition, 44, 1401-1404. https://doi.org/10.1002/anie.200462114

[103] Mitchell, N. and Howorka, S. (2008) Chemical Tags Facilitate the Sensing of Individual DNA Strands with Nanopores. Angewandte Chemie International Edition, 47, 5565-5568. https://doi.org/10.1002/anie.200800183

[104] Xie, P., Xiong, Q., Fang, Y., Qing, Q. and Lieber, C.M. (2012) Local Electrical Potential Detection of DNA by Nanowire-Nanopore Sensors. Nature Nanotechnology, 7, 119-125. https://doi.org/10.1038/nnano.2011.217

[105] Stefan, W.K., Alexander, Y.G., Yitzhak, R. and Cees, D. (2011) Modeling the Conductance and DNA Blockade of Solid-State Nanopores. Nanotechnology, 22, Article ID: 315101. https://doi.org/10.1088/0957-4484/22/31/315101

[106] Lagerqvist, J., Zwolak, M., and Di Ventra, M. (2007) Influence of the Environment and Probes on Rapid DNA Sequencing via Transverse Electronic Transport. Biophysical Journal, 93, 2384-2390. https://doi.org/10.1529/biophysj.106.102269

[107] Krstic, P.S., Wells, J.C., Fuentes-Cabrera, M., Xu, D. and Lee, J.W. (2007) Toward Electronic Conductance Characterization of DNA Nucleotide Bases. Solid State Phenomena, 121-123, 1387-1390. https://doi.org/10.4028/www.scientific.net/SSP.121-123.1387

[108] Tsutsui, M., Taniguchi, M., Yokota, K. and Kawai, T. (2010) Identifying Single Nucleotides by Tunnelling Current. Nature Nanotechnology, 5, 286-290. https://doi.org/10.1038/nnano.2010.42

[109] Chang, S., Huang, S., He, J., Liang, F., Zhang, P., Li, S., Chen, X., Sankey, O. and Lindsay, S. (2010) Electronic Signatures of all Four DNA Nucleosides in a Tunneling Gap. Nano Letters, 10, 1070-1075. https://doi.org/10.1021/nl1001185

[110] Huang, S., He, J., Chang, S., Zhang, P., Liang, F., Li, S., Tuchband, M., Fuhrmann, A., Ros, R. and Lindsay, S. (2010) Identifying Single Bases in a DNA Oligomer with Electron Tunneling. Nature Nanotechnology, 5, 868-873. https://doi.org/10.1038/nnano.2010.213

[111] Ohshiro, T., Tsutsui, M., Matsubara, K., Furuhashi, M., Taniguchi, M. and Kawai, 
T. (2012) Single-Molecule Tunnel-Current Based Identification of DNA/RNA Towards Sequencing by Using Nano-MCBJ. 16th International Conference on Miniaturized Systems for Chemistry and Life Sciences, Okinawa, Japan, 28 October-1 November 2012, 204-206.

[112] Cheng, L. (2016) Role of Hydrogen Bonding in the Formation of Adenine Chains on $\mathrm{Cu}(110)$ Surfaces. Materials, 9, 1016-1027. https://doi.org/10.3390/ma9121016

[113] Kestell, J., Boscoboinik, J.A., Cheng, L., Garvey, M., Bennett, D.W. and Tysoe, W.T. (2015) Structural Changes in Self-Catalyzed Adsorption of Carbon Monoxide on 1,4-Phenylene Diisocyanide Modified Au(111). Journal of Physical Chemistry C, 119, 18317-18325. https://doi.org/10.1021/acs.jpcc.5b04783

[114] Nelson, B.P., Grimsrud, T.E., Liles, M.R., Goodman, R.M. and Corn, R.M. (2000) Surface Plasmon Resonance Imaging Measurements of DNA and RNA Hybridization Adsorption onto DNA Microarrays. Analytical Chemistry, 73, 1-7. https://doi.org/10.1021/ac0010431

[115] Xu, W. (2008) Adsorption of Organic Molecules on Solid Surfaces. A Scanning Tunneling Microscopy Study. PhD Thesis, Interdisciplinary Nanoscience Center (iNANO) and Department of Physics and Astronomy University of Aarhus, Denmark.

[116] Sowerby, S.J., Edelwirth, M. and Heckl, W.M. (1998) Self-Assembly at the Prebiotic Solid-Liquid Interface: Structures of Self-Assembled Monolayers of Adenine and Guanine Bases Formed on Inorganic Surfaces. The Journal of Physical Chemistry B, 102, 5914-5922. https://doi.org/10.1021/jp9806841

[117] Schöning, M. and Poghossian, A. (2006) Bio FEDs (Field-Effect Devices): State-ofthe-Art and New Directions. Electroanalysis, 18, 1893-1900. https://doi.org/10.1002/elan.200603609

[118] Lee, C.-S., Kim, S.K. and Kim, M. (2009) Ion-Sensitive Field-Effect Transistor for Biological Sensing. Sensors, 9, 7111-7131. https://doi.org/10.3390/s90907111

[119] Pijanowska, D.G. and Torbicz, W. (2005) Biosensors for Bioanalytical Applications. Bulletin of the Polish Academy of Sciences. Technical Sciences, 53, 251-260.

[120] Kimura, J., Ito, N., Kuriyama, T., Kikuchi, M., Arai, T., Negishi, N. and Tomita, Y. (1989) J. Electrochem. Soc., 136, 1744-1747.

[121] Bergveld, P. (1970) Development of an Ion-Sensitive Solid-State Device for Neurophysiological Measurements. IEEE Transactions on Biomedical Engineering, BME-17, 70-71. https://doi.org/10.1109/TBME.1970.4502688

[122] Veigas, B., Fortunato, E. and Baptista, P.V. (2015) Field Effect Sensors for Nucleic Acid Detection: Recent Advances and Future Perspectives. Sensors, 15, 10380-10398. https://doi.org/10.3390/s150510380

[123] Hafeman, D.G., Parce, J.W. and McConnel, H.M. (1988) Light-Addressable Potentiometric Sensor for Biochemical Systems. Science, 240, 1182-1185. https://doi.org/10.1126/science.3375810

[124] Gasparyan, F., Zadorozhny, I., Khondkaryan, H., Arakelyan, A. and Vitusevich, S. (2018) Photoconductivity, pH Sensitivity, Noise, and Channel Length Effects in Si Nanowire FET Sensors. Nanoscale Research Letters, 13, 87-96. https://doi.org/10.1186/s11671-018-2494-5

[125] Estrela, P., Stewart, A., Yan, F. and Migliorato, P. (2005) Field Effect Detection of Biomolecular Interactions. Electrochimica Acta, 50, 4995-5000.

https://doi.org/10.1016/j.electacta.2005.02.075

[126] Khan, M.I., Khan, A.M., Nouman, A., Azhar, M.I. and Saleem, M.K. (2012) pH 
Sensing Materials for MEMS Sensors and Detection Techniques. Int. Conf. on Solid-State and Integrated Circuit (ICSIC 2012) IPCSIT, Vol. 32, IACSIT Press, Singapore, 18-23. http://www.ipcsit.com/vol32/004-ICSIC2012-D0022.pdf

[127] Bergveld, P. (2003) Thirty Years of ISFETOLOGY: What Happened in the Past 30 Years and What May Happen in the Next 30 Years. Sensors and Actuators B: Chemical, 88, 1-20. https://doi.org/10.1016/S0925-4005(02)00301-5

[128] Caras, S. and Janata, J. (1980) Field Effect Transistor Sensitive to Penicillin. Analytical Chemistry, 52, 1935-1937. https://doi.org/10.1021/ac50062a035

[129] Lee, C.H., Seo, H.I., Lee, Y.C., Cho, B.W., Jeong, H. and Dohn, B.K. (2000) All Solid Type ISFET Glucose Sensor with Fast Response and High Sensitivity Characteristics. Sensors and Actuators B: Chemical, 64, 37-41. https://doi.org/10.1016/S0925-4005(99)00480-3

[130] Sekiguchi, T., Nakamura, M., Kato, M., Nishikawa, K., Hokari, K., Sugiyama, T. and Asaka, M. (2000) Immunological Helicobacter pylori Urease Analyzer Based on Ion-Sensitive Field Effect Transistor. Sensors and Actuators B: Chemical, 67, 265-269. https://doi.org/10.1016/S0925-4005(00)00522-0

[131] Offenhäusser, A., Sprössler, C., Matsuzawa, M. and Knoll, W. (1997) Field-Effect Transistor Array for Monitoring Electrical Activity from Mammalian Neurons in Culture. Biosensors and Bioelectronics, 12, 819-826.

https://doi.org/10.1016/S0956-5663(97)00047-X

[132] Meyburg, S., Goryll, M., Moers, J., Ingebrandt, S., Böcker-Meffert, S., Lüth, H. and Offenhäusser, A. (2006) N-Channel Field-Effect Transistors with Floating Gates for Extracellular Recordings. Biosensors and Bioelectronics, 21, 1037-1044. https://doi.org/10.1016/j.bios.2005.03.010

[133] Chang, J.C. and Kan, Y.W. (1979) Beta 0 Thalassemia, a Nonsense Mutation in Man. Proceedings of the National Academy of Sciences of the USA, 76, 2886-2889. https://doi.org/10.1073/pnas.76.6.2886

[134] Schöning, M.J. and Poghossian, A. (2002) Recent Advances in Biologically Sensitive Field-Effect Transistors (BioFETs). Analyst, 127, 1137-1151. https://doi.org/10.1039/B204444G

[135] Hu, Y. (2015) Advanced Sensing and Processing Methodologies for ISFET Based DNA Sequencing. A Report Submitted for the Degree of Doctor of Philosophy of Imperial College London, London.

[136] Kaisti, M., Kerko, A., Aarikka, E., Saviranta, P., Boeva, Z., Soukka, T. and Lehmusvuori, A. (2017) Real-Time Wash-Free Detection of Unlabeled PNA-DNA Hybridization Using Discrete FET Sensor. Scientific Rpeorts, 7, Article No. 15734. https://doi.org/10.1038/s41598-017-16028-7

[137] Purushothaman, S., Toumazou, C. and Ou, C.-P. (2006) Protons and Single Nucleotide Polymorphism Detection: A Simple Use for the Ion Sensitive Field Effect Transistor. Sensors and Actuators B: Chemical, 114, 964-968. https://doi.org/10.1016/j.snb.2005.06.069

[138] Russel, W.B., Saville, D.A. and Schowalter, W.R. (1989) Colloidal Dispersions. Cambridge University Press, Cambridge. https://doi.org/10.1017/CBO9780511608810

[139] Fologea, D., Uplinger, J., Thomas, B., McNabb, D.S. and Li, J. (2005) Slowing DNA Translocation in a Solid-State Nanopore. Nano Letters, 5, 1734-1737. https://doi.org/10.1021/nl0510630

[140] Peng, H.B. and Ling, X.S.S. (2009) Reverse DNA Translocation through a Sol- 
id-State Nanopore by Magnetic Tweezers. Nanotechnology, 20, Article ID: 185101. https://doi.org/10.1088/0957-4484/20/18/185101

[141] He, Y., Tsutsui, M., Fan, C., Taniguchi, M. and Kawai, T. (2011) Controlling DNA Translocation through Gate Modulation of Nanopore Wall Surface Charges. ACS Nano, 5, 5509-5518. https://doi.org/10.1021/nn201883b

[142] Akenson, M., Branton, D., Kasianowicz, J.J., Brandin, E. and Deamer, D.W. (1999) Microsecond Time-Scale Discrimination Among Polycytidylic Acid, Polyadenylic Acid, and Polyuridylic Acid as Homopolymers or as Segments Within Single RNA Molecules. Biophysical Journal, 77, 3227-3233.

https://doi.org/10.1016/S0006-3495(99)77153-5

[143] Maitra, R.D., Kim, J. and Dunbar, W.B. (2012) Recent Advances in Nanopore Sequencing. Electrophoresis, 33, 3418-3428.

https://doi.org/10.1002/elps.201200272

[144] Pyykkö, P. and Atsumi, M. (2009) Molecular Double-Bond Covalent Radii for Elements Li-E112. Chemistry: A European Journal, 15, 12770-12779. https://doi.org/10.1002/chem.200901472

[145] Han, M.Y. (2010) Electronic Transport in Graphene Nanoribbons. Columbia University Press, $\mathbb{N e w}$ York.

[146] Gasparyan, L., Mazo, I., Simonyan, V. and Gasparyan, F. (2019) Modified DNA Sequencing Method through Effective Regulation of the DNA Translocation Speed in Aqueous Solution. Proceedings of International Conference on Advances in Functional Materials, The George Washington University, Washington DC, 22-24 July 2019, in Press. 